\title{
The Creative Synthesis and the Global Psychology
}

\author{
Luca Tateo $^{1}$ (D) - Giuseppina Marsico ${ }^{2,3}$
}

Published online: 2 March 2021

(c) The Author(s), under exclusive licence to Springer Nature Switzerland AG part of Springer Nature 2021

\section{A Developmental Process}

The past year, 2020, has undoubtedly been one of the longest, most complex, and difficult years that mankind has remembered for a long time now. The team of Human Arenas expresses strong compassion, proximity, and condolence to all the victims of the pandemic and all the people who have been affected in many ways worldwide. But we also look with hope at the many forms of development and creative resistance that mankind can generate in difficult times. It has also been a period of development for which the crisis is the complementary side. The academic work has been of course affected by the consequences of the global pandemic crisis, as any other human activity. Yet, once again, academia has been a privileged area and our work has been touched but not completely disrupted. Despite the many problems, we have had the possibility to continue our work with Human Arenas. Our many authors, associates, and reviewers have continued to produce high quality and innovative ideas. Human Arenas has indeed achieved new successes like the inclusion into the Scopus list of sources. The process will be visible in the Spring 2021 and will be retroactive to all the volumes since the creation of the journal. Indexing is not only an acknowledgement of the work done by our editorial team and by the publisher's team, but also a new great responsibility for all of us. During the year 2020, Human Arenas had 38,371 article downloads, 150 submissions, 87 accepted papers, and 68 published papers. Our figures are constantly growing and we consider the Scopus indexing as a new starting point for continuing the development of our collaborative construction of knowledge. As usual, we express our deep gratitude not only to the authors but also to the reviewers in the acknowledgement that we publish in the first issue of each volume. The dialogical review process is indeed the engine of the ideas' cultivation process that we want to achieve on Human Arenas (Tateo \& Marsico, 2020).

Luca Tateo

luca.tateo@isp.uio.no

1 University of Oslo, Oslo, Norway

2 University of Salerno, Fisciano, SA, Italy

3 Federal University of Salvador da Bahia, Salvador, BA, Brazil 


\section{The Creative Synthesis}

The year 2020 has also been the anniversary of the death of Wilhelm Wundt (1832-1920). We see a form of synchronicity in celebrating the anniversary of one of the most relevant thinkers in the study of the relationship between individual consciousness and the collective activity (Tateo \& Iannaccone, 2012) in the same year of the Covid-19 pandemic. The pandemic has made clear that the future existence of mankind will be punctuated by new forms of global crises that psychology can no longer address as mere sum of individual distress. Wundt's thought has been for long time oversimplified and bent to the needs of an individualist and positivist psychology. The complexity of his thinking has been neglected because psychology often tends to abhor complexity, falling into what Wundt called "Vulgärpsychologie" (Diriwächter, 2021, p. 6). In the first issue of the 2021, we publish a special section to recall and elaborate Wundt's ideas and unexplored avenues. The contribution of Diriwächter (2021) and Jovanović (2021) provides a thorough contextualization of Wundt's work, discussing the whole and the offspring of his ideas, such as the two Leipzig's schools, which have been selectively forgotten by psychology. The contributions provide complementary views that make visible the complex image of a scholar who set most of the fundamental questions of psychology. "Wundt should be well remembered by psychologists because the general problems he saw for psychological science have never disappeared" (Fierro \& de Freitas Araujo, 2020, in this issue).

Wundt's philosophy of psychology provides the creative synthesis between the study of the individual and the collective forms of thinking and acting understood as wholes. From the articles published in this issue, we discover that the concern of posing the wholes and their developmental features as object of psychology do not match the elementist and associationist version ascribed to Wundt by the following vulgata of positivistic and individualistic psychology. This is not only a matter of history of psychology. We believe that the current Covid-19 pandemic is the specimen of a new type of challenge for psychology (Dege \& Strasser, 2021). We need to understand the psychic experience as a complex whole-a combination of cognition, emotion and will in Wundt's words-which is itself part of a whole of individual and collective. In this sense, psychology must still "provide an answer to the question of the relation between the individual and the world (or culture). When looking for an answer though, psychology still seems to lack addressing how to go beyond its individual centric analyses." (Rodax \& Benetka, 2021, in this issue).

Yet, the pandemic also represents the proof that mankind must no longer think itself as separate from a natural realm. Human exceptionalism falls short before the evidence that we the humans are part of an ecosystemic whole. Individual psychology, merely understood as human exceptionalism, was meaningless for Wundt as it is still meaningless for us today. What are the implications of taking the idea of the wholeness (Ganzheitspsychologie) in the psychological investigation seriously? The idea of the whole implies that any part of a bounded field is not equal to the field as a whole. Nevertheless, this is still a static depiction. According to Valsiner (2004): "Ganzheitsthinking requires the dynamic nature of the holistic phenomena. The structure of the field is in constant motion, or its stationary moments are in tension towards motions" (p. 86, original italic). There is not such a fixed entity as a field at given time but only Ganzheits in motion. This has particular significance if we really want to capture the complexity of human phenomena like for instance the Covid-19 pandemic and its evolutionary process. Ganzheitspsychologie offers the essential tools to study complex, 
fluid, and self-transforming phenomena. The contemporary psychological science needs to refocus on the multi-level structure of holistic fields where the structure is fluid and fluidity supports the structures.

Wundt's legacy is relevant for current psychology also in other respects. First, he was an integral humanist. His background was in medicine as well as in philosophy, and he was learned in humanities. He was appointed as professor in anthropology and medical psychology (Klempe, 2021). Psychology nowadays overlooks the importance of the humanistic education, promoting a restricted and hyperspecialized technical training that impoverishes the sensitivity and intellectual curiosity of the students. Remembering Wundt means to appreciate once again the complexity of the intellectual endeavour of its scholarship, in which the quest for the understanding of human mind and culture produced an ethical stance of high profile. Rodax and Benetka (2021) illustrate this attitude reminding Wundt's letter to his former mentee and later intellectual competitor Oswald Külpe. In 1907, Wundt wrote that he was "absolutely convinced that one could disagree in questions of science and even that it is possible to run in a fundamentally different direction, without harming the feelings of personal friendship and sincere scientific respect" (quoted in Rodax $\&$ Benetka, 2021, p. 4). Such an ethical attitude toward intellectual exchange is no longer current currency in the hypercompetitive and belligerent academic context. Young scholars are told that they shall care about their restricted field of interest and compete arduously against those who may override them in the publish or perish rat race.

\section{What Is Coming}

The Covid-19 pandemic has woken up scholars from a sort of neo-liberalist sleepiness and revealed the need of a new ethical stance. The pandemic is not the same for everyone, and inequalities appeared even more clearly. The complex of groups and activities that we call science can no longer be conceived as a form of anthropic domination and exploitation of an alien nature. Science is at the same time the existential dread of our human consciousness and the way we can acknowledge our being part of the whole ecosystem. At the same time, the joint effort of scholars worldwide, the web of daily connections we all have established and maintained through virtual conference rooms, and the meetings at any unlikely hours of the day or night have created a new sense of solidarity and proximity. Many initiatives have flourished, and Human Arenas will account for some of them in the forthcoming issues. In 2021, we will publish a special section called Arena of crisis, edited by Martin Dege and Irene Strasser, which originates from the virtual conference The Psychology of Global Crises, organized in May 2020 by the American University in Paris. Scholars and artists from different disciplines met to discuss the complexity of the global crises and how mankind can use this opportunity to develop new forms of awareness, reflexivity, and critical practices. Human Arenas is very happy to open this thread of discussion by publishing contributions over consecutive issues. In the same vein, the journal will publish another special section called Arena of pandemic. The section focuses on the meaning of the pandemic on the global ecosystem, including human activities and lives, both at the individual and collective level. Scholars from all the continents and different disciplines engage in a discussion that tries to overcome the limited interpretation in terms of trauma and resilience, focusing on the consequences, future directions, and emerging phenomena related to the pandemic. 


\section{Conclusion: Global Psychology}

The vision of Human Arenas for 2021 is to promote the resurgence of a global psychology, which is a study of the human condition and psychological experience as a whole and as a part of a larger ecosystemic whole. We believe this could give a new sense to the concept of Ganzheitspsychologie: an epistemological stance that do not conceives phenomena in terms of correlations between variables but in terms of dynamic configurations unfolding in a developmental transformation. Such a global psychology developed by integral humanists is very close to Wundt's scholarship. Leipzig's laboratory was not just a centre of experimentation, as most of the partial accounts in the mainstream psychology textbook report (Jovanović, 2021). It was also an intellectual hub where scholars from different countries and different theoretical perspectives gathered to develop innovative ideas. The Covid-19 pandemic has clearly showed the limits of a national and nationalistic take on the university. Psychology is still rooted into a Humboldtian model of university (Nybom, 2003), in which science and national identity feed into each other and cannot but fall short before the global challenges. In its fourth year of life, Human Arenas aims at promoting an intense intercultural and interdisciplinary dialogue.

\section{References}

Dege, M., \& Strasser, I. (Eds.). (2021). Global pandemics and epistemic crises in psychology. A sociophilosophical approach. Routledge.

Diriwächter, R. (2021). Remembering Wilhelm Wundt and the Second Leipzig School of Psychology. $H u$ Arenas. https://doi.org/10.1007/s42087-020-00158-y.

Fierro, C., \& de Freitas Araujo, S. (2020). A case for a philosophical history of psychology: An interview with Saulo de Freitas Araujo at the centenary of the death of Wilhelm Wundt (1832-1920). Human Arenas. https://doi.org/10.1007/s42087-020-00165-z.

Klempe, S. H. (2021). The importance of Leibniz for Wundt. Hu Arenas. https://doi.org/10.1007/s42087-02000169-9.

Jovanović, G. (2021). How psychology repressed its founding father Wilhelm Wundt. Human Arenas

Nybom, T. (2003). The Humboldt Legacy: Reflections on the past, present, and future of the European University. Higher Education Policy, 16, 141-159. https://doi.org/10.1057/palgrave.hep.8300013.

Rodax, N., \& Benetka, G. (2021). Debating experimental psychology's frontiers: Re-discovering Wilhelm Wundt's contribution to contemporary psychological research. Hu Arenas. https://doi.org/10.1007/ s42087-020-00173-z.

Tateo, L., \& Iannaccone, A. (2012). Social representations, individual and collective mind: A study of Wundt, Cattaneo and Moscovici. Integrative Psychological and Behavioral Science, 46(1), 57-69. https://doi.org/10.1007/s12124-011-9162-y.

Tateo, L., \& Marsico, G. (2020). Reviewing as dialogical development of ideas. Human Arenas, 3, 1-5. https://doi.org/10.1007/s42087-020-00100-2.

Valsiner, J. (2004). What to do with the whole? Implications from taking Ganzheitspsychologie seriously. From Past to Future, Vol. 5(1), (p.85-87).

Publisher's Note Springer Nature remains neutral with regard to jurisdictional claims in published maps and institutional affiliations. 